\title{
Mechanical characterization of sequentially layered photo-clickable thiol-ene
}

\section{hydrogels}

Aaron H. Aziz, ${ }^{1,2}$ Joseph Wahlquist, ${ }^{3}$ Aaron Sollner, ${ }^{1}$ Virginia Ferguson, ${ }^{2,3,5}$ Frank W. DelRio, ${ }^{4}$ Stephanie J.

Bryant $t^{1,2,5}$

${ }^{1}$ Department of Chemical and Biological Engineering, University of Colorado, UCB 596, Boulder, C0, 80309, USA

${ }^{2}$ BioFrontiers Institute, University of Colorado, Boulder, CO, USA

${ }^{3}$ Department of Mechanical Engineering, University of Colorado, Boulder, CO, USA

${ }^{4}$ Applied Chemicals and Materials Division, Material Measurement Laboratory, National Institute of Standards and Technology, Boulder, C0, 80305, USA

${ }^{5}$ Material Science \& Engineering Program, University of Colorado, Boulder, CO, USA

\section{Corresponding Author}

Name: Stephanie J. Bryant

Department: Chemical and Biological Engineering

Address: 3415 Colorado Ave, Boulder, CO 80309-0596

Telephone: 303-735-6714

Fax: 303-492-8425

E-mail: stephanie.bryant@colorado.edu 


\begin{abstract}
Multi-layer hydrogels are promising for tissue engineering due to the ability to control the local properties within each layer. However, the interface that forms between each layer has the potential to affect the performance of the hydrogel. The goals of this study were to characterize how the interface forms via its thickness and mechanical properties, identify its impact on the overall hydrogel properties, and provide new insights into how to control the interface. A photo-clickable poly(ethylene glycol) hydrogel was used to form bilayer hydrogels that were sequentially polymerized in a step-and-repeat process. Different processing conditions were studied: the time (0-20 min) before initiating polymerization of the second layer (soak time, $\mathrm{t}_{\mathrm{s}}$ ) and the hydrogel crosslink density (the same, less crosslinked, or more crosslinked) of the first layer as compared to the second layer. Interface thickness was characterized by confocal microscopy, monomer transport by Fickian diffusion, single and bilayer hydrogel mechanics by bulk moduli measurements, and interface moduli measurements using AFM, nanoindentation, and strain mapping. The interface thickness ranged from $\sim 70$ to $600 \mu \mathrm{m}$ (1-10\% of total height) depending on processing conditions, but did not affect the bulk hydrogel modulus. Analysis of monomer transport revealed that convection, due to changes in hydrogel swelling, and diffusion contribute to interface thickness. Nanomechanical analysis of bilayer hydrogels formed from soft ( $75 \mathrm{kPa})$ and stiff (250 kPa) layers showed a gradient in elastic modulus across the interface, which corresponded to strain maps. In summary, this work identifies that diffusive and convective transport of monomers across the interface controls its thickness and that a mechanically robust interface forms, which does not affect the hydrogel modulus. By controlling the processing conditions, the thickness of the interface can be tuned without affecting the mechanical properties of the bulk hydrogel.
\end{abstract}

Keywords: multi-layer hydrogel, interfacial properties, nanoindentation, atomic force microscopy 


\section{Introduction}

Many tissues in the body exist as multi-layer structures with each layer having distinct biochemical and mechanical properties that together contribute to the overall function of the tissue. Examples of such tissues include cartilage, which is composed of superficial, middle and deep zonal layers, skin, which consists of an epidermis and dermis layer, and the junction that connects cartilage to bone or tendons and ligaments to bone. Between each layered structure, an interface forms that can be characterized by either extended or abrupt gradients in the biochemical and/or mechanical properties. For example, abrupt gradients are present in the mechanical transition at the osteochondral junction between stiff calcified cartilage and compliant hyaline cartilage (Campbell et al., 2012) and in the compositional transition across the muscle-tendon junction (Ker, 2007). The ability to mimic these complex tissue structures in a scaffold is an important step towards developing successful strategies for tissue engineering composite tissues.

Multi-layered hydrogels can be fabricated such that their chemistry and properties (e.g., crosslinking and porosity) vary in space. Mild polymerization conditions coupled with a high water content make hydrogels particularly suitable for the encapsulation and culture of cells in 3D scaffolds (Nicodemus and Bryant, 2008) As such, multi-layer hydrogels are being designed and investigated for tissue engineering of complex multistructured tissues. For example, multi-layer fibrous hydrogels made from chitosan were designed where keratinocytes were seeded into a layer simulating the dermis, fibroblasts seeded into a second layer representing the protective epidermis, and a thin film to mimic the basement membrane to engineer a skin substitute (Lin et al., 2015). This tri-layered hydrogel enabled both cell types to replicate the striation of full thickness skin more accurately and in a shorter time than single or bi-layer counterparts. In a separate study, a multi-layer hydrogel formed from crosslinked poly(ethylene glycol) (PEG) was tuned by introducing different extracellular matrix molecules into each layer to mimic the chemical makeup of the different zones in cartilage (Nguyen et al., 2011). In doing so, 3D hydrogel niches were created that allowed a single population of stem cells to differentiate into zone-specific chondrocytes. In another study, the composition and mechanical properties in each layer of a crosslinked PEG hydrogel was varied to capture the physiochemical cues that arise in osteochondral tissues under mechanical loads (Steinmetz et al., 2015). 
When human mesenchymal stem cells were encapsulated in the multi-layer hydrogel and subjected to dynamic loading, differentiation towards a chondrogenic phenotype was observed in one layer and an osteogenic phenotype was observed in the other layer. Taken together, these examples among others demonstrate that multi-layered hydrogels are promising for tissue engineering complex multi-layer composite tissues.

In forming multi-layer hydrogels, the interface that forms between two adjacent layers is critical to the overall function of the hydrogel. For example, if the layers are not well integrated and secured together, the interface is prone to shear failure and can lead to delamination (Kandel et al., 2006; Sherwood et al., 2002). For interfaces with abrupt gradients, the two dissimilar materials disrupt strain transfer and can lead to areas of high stress concentration that result in failure at the interface (Erdogan, 1995). On the contrary, if the interface that forms is large, the local biochemical and mechanical properties can influence the fate of cells embedded within this region (Steinmetz et al., 2015). Thus, controlling the interface is important to designing multi-layer hydrogels that recapitulate extended or abrupt gradients found in native interfaces and that lead to mechanically robust interfaces to minimize failure. The resulting interface when multi-layer hydrogels are formed has received little attention.

This study investigates a bi-layer PEG hydrogel and the interface that forms between the two layers. PEG hydrogels were chosen for their promise in producing multi-layer hydrogels for use in vascular (Fischer et al., 2015; Shinohara et al., 2013) and musculoskeletal (Fuoco et al., 2012; Hwang et al., 2010; Lin et al., 2014; Nguyen et al., 2012; Paxton et al., 2009; Steinmetz et al., 2015) tissue engineering. The objectives for this study are to characterize how the interface forms via its thickness and mechanical properties, identify its impact on the overall hydrogel properties, and provide new insights into how to control the interface. Bilayer PEG hydrogels were sequentially polymerized from photo-clickable thiol-ene macromolecular monomers. This reaction scheme was chosen for its promise in tissue engineering (Fairbanks et al., 2009; Tibbitt and Anseth, 2009) and its highly specific, efficient, and rapid reaction (Hoyle and Bowman, 2010). Specifically, this study investigated (a) the effects of monomer transport between the two layers as a function of processing conditions on interface thickness and bulk mechanical properties and (b) the local mechanical 
properties and strain transfer across the interface when each hydrogel layer is formed with different mechanical properties. The latter is important for applications where mechanical forces are applied to multilayer hydrogels. Overall, this study provides new insight into how processing parameters influence the formation and properties of the interface and ultimately the contribution that the interface has on the macroscopic properties. Findings from this study will aid the development of multi-layer hydrogels where the interface can be tuned to create thin interfaces that lead to abrupt property changes across the interface or thick interfaces where large property gradients across the interface are required.

\section{Materials and Methods}

\subsection{Monomer synthesis}

8-arm PEG with terminal amines (20,000 g/mol; JenKem Technology USA, Plano, TX) was functionalized with norbornenes by reacting 5-norbornene-2-carboxcylic acid (Sigma-Aldrich, St. Louis, MO) with 2-(1H-7azabenzotriazol-1-yl)-1,1,3,3-tetramethyl uranium hexafluorophosphate methanaminium (HATU) (ChemImpex International, Inc., Wool Dale, IL), and N,N-diisopropylethylamine (DIPEA) (Chem-Impex) in dimethylformamide (DMF)/dichloromethane (DCM) (Sigma-Aldrich). The reaction was allowed to proceed overnight at room temperature under an inert atmosphere. The product was precipitated in diethyl ether (Sigma-Aldrich), filtered, dialyzed, and lyophilized. The extent of conjugation of norbornene to each arm of the 8 -arm PEG-amine was determined to be $92 \%$ using ${ }^{1} \mathrm{H}$ NMR by comparing the protons across the carboncarbon double bond in the norbornene to the methylene protons in PEG.

\subsection{Fabrication of PEG Hydrogels}

A precursor solution consisting of 8-arm PEG-norbornene (PEGnor) monomer, PEG-dithiol (PEGdt) crosslinker (1000 or $3400 \mathrm{~g} / \mathrm{mol}$; Sigma-Aldrich) at 1:1 thiol:ene ratio (assuming 100\% norbornene conjugation), and $0.05 \%(\mathrm{w} / \mathrm{w})$ photoinitiator, 1-(4-(2-Hydroxyethoxy)-phenyl)-2-hydroxy-2-methyl-1propane-1-one (I2959; Ciba Specialty Chemicals, Tarrytown, NY), in deionized water ( $\left.\mathrm{diH}_{2} \mathrm{O}\right)$ was photopolymerized by ultraviolet light (10 minutes, 5-10 mW/cm², $352 \mathrm{~nm}$ ). The concentration of 8-arm PEGnorbornene varied by 10, 15 or $25 \%$ (w/w) (Hydrogel I, II/III and IV respectively) depending on the 
experiment. Hydrogels were formed in either a cylindrical or rectangular geometry depending on the experiment (as described below). For single layer constructs, full height (i.e., $5 \mathrm{~mm}$ ) hydrogels were fabricated from a single macromer solution. For bilayer constructs, half-height (i.e., $2.5 \mathrm{~mm}$ ) hydrogels were fabricated from one macromer solution that was polymerized to form the first layer. Previous studies have reported that complete polymerization of similar precursor formulations is reached in less than a minute (Roberts and Bryant, 2013) and therefore ten minutes ensures that the first layer has polymerized completely before introducing the first layer. Following polymerization of the first layer, a second macromer solution was carefully deposited on top of the first layer to form the second layer and to reach full height (i.e., $5 \mathrm{~mm}$ ). The second solution was left to allow for transport of the monomers into the first hydrogel layer for prescribed periods of time $\left(\mathrm{t}_{\mathrm{s}}\right)$, after which the second layer was polymerized. A schematic representation of the fabrication process is shown in Fig. 1A.

\subsection{Swelling Studies and Hydrogel Characterization}

Single layer, full height cylindrical hydrogels prepared as stated above were weighed immediately after polymerization and then allowed to swell in $\mathrm{diH}_{2} \mathrm{O}$ for 48 hours at room temperature. Equilibrium swollen hydrogels ( $n=3$ /group) were weighed to determine the equilibrium swollen mass. The dry polymer mass was obtained for each hydrogel after lyophilization. The equilibrium mass swelling ratio, $q$, was calculated by dividing the equilibrium swollen mass by the polymer dry mass. The equilibrium swelling ratio, $Q$, was estimated using densities of the respective polymer and solvent by

$$
Q=1+\frac{\rho_{p}}{\rho_{s}}(q-1)
$$

Here $\rho_{p}$ is the density of the polymer, which for PEG is assumed $1.07 \mathrm{~g} / \mathrm{mL}$, and $\rho_{s}$ is the density of the solvent, which in this case for water is $1 \mathrm{~g} / \mathrm{mL}$. The hydrogel mesh size, $\xi$, was calculated by the following equation (Canal and Peppas, 1989),

$$
\xi=v_{2, s}^{-1 / 3} l C_{n}^{1 / 2} n^{1 / 2}
$$

where $v_{2, s}$ is the equilibrium polymer volume fraction in the gel (i.e., inverse of the swelling ratio, $Q$ ), $l$ is the average bond length, which for PEG is $l=1.49 \AA, C_{n}$ is the characteristic ratio of the polymer, which is assumed to be 4.0 (Merrill et al., 1993), and $n$ is the number of bonds between the crosslinks, which was 
determined from the crosslink density. The crosslink density and the polymer-solvent interaction $\left(\chi_{12}\right)$ parameter were estimated using a self-learning algorithm (Akalp et al., 2015) that combines Flory-Rehner theory with theories of mixture and poroelasticity (Flory and Erman, 1982; Flory, 1953; Holmes and Mow, 1990; Peppas, 1986). The crosslink density was determined to be $0.020,0.026,0.032$, and $0.059 \mathrm{M}$ and $\chi_{12}$ was determined to be $0.488,0.451,0.489$, and 0.486 for Hydrogels I, II, III, and IV respectively.

\subsection{Interfacial Thickness Measurement}

Rectangular PEG hydrogels (5 mm height, $5 \mathrm{~mm}$ width, $1 \mathrm{~mm}$ thick) were made following methods described above. Fluorescently labeled hydrogels were formed by incorporating a fluorophore into each layer. In brief, 0.01 mM Alexa Fluor ${ }^{\circledR} 488$ C5 Maleimide (Thermo Fisher Scientific, Waltham, MA) was added to one precursor solution and $0.01 \mathrm{mM}$ Alexa Fluor® 546 C5 Maleimide (Thermo Fisher Scientific) was added to the second precursor solution prior to polymerization leading to the formation of a dual-fluorescent bilayer hydrogel. Hydrogels were imaged by confocal microscopy (Zeiss LSM 5 Pascal system using a Zeiss Axiovert microscope). Interface thickness was determined from confocal microscopy images and NIH Image J software. In brief, a line was manually drawn perpendicular to the interface and intensity along the distance of the line was determined for each of the fluorophore channels. The total distance across which both fluorophore channels detected fluorescence signal over the background denoted the interface thickness.

\subsection{Mechanical Testing of PEG Hydrogels}

Cylindrical PEG hydrogels ( $5 \mathrm{~mm}$ height and $5 \mathrm{~mm}$ diameter) were made following methods described above. Compressive testing was conducted on a Mechanical Testing System (MTS; Eden Prairie, MN). Equilibrium swollen hydrogels ( $n=3$ /group) were tested in unconfined compression on dry platens with minimal sticking at a constant strain rate of $0.5 \mathrm{~mm} / \mathrm{min}$ to $50 \%$ strain $(2 \mathrm{mN}$ pre-load; limited by a $9 \mathrm{~N}$ max load with a total load cell capacity of $10 \mathrm{~N}$ ). The tangent compressive modulus was calculated from the slope of the stressstrain curve at low (10-15\%) strain and high (40-45\%) strain.

\subsection{One-Dimensional Diffusion Studies}


Rectangular PEG hydrogels (10 mm width, $25 \mathrm{~mm}$ length, $1 \mathrm{~mm}$ thick) were made between two glass slides following polymerization methods described above. The hydrogel was maintained between the two glass slides and reservoirs on either end of the hydrogel were filled with $\mathrm{diH}_{2} \mathrm{O}$. Hydrogels were allowed to swell for 48 hours at room temperature. Pre-swollen hydrogels were placed in a VersaDoc MP 4000 Molecular Imaging System (Bio-Rad; Hercules, CA) and one reservoir was replaced with either an aqueous solution of 0.01 mM Alexa Fluor® 488 C5 Maleimide pre-reacted with 10 mM 8-arm PEG-thiol (20,000 g/mol; JenKem Technology USA, Plano, TX) or 0.01 mM Alexa Fluor® 488 C5 Maleimide pre-reacted with 100 mM methoxy PEG-thiol (800 MW; Sigma-Aldrich). Images were acquired at 12, 39, and 46 hours for the 8-arm PEG molecule and at 24 and 30 hours for the methoxy PEG-thiol molecule. From these images, Image J was used to plot concentration profiles of fluorescence intensity as a function of distance into the gel. These profiles were fit to the solution of Fick's second law in one dimension via the curve fitting tool in MATLAB (The Mathworks, Inc., Natick, MA) to extract approximate diffusivities for the hydrogel conditions explored.

\subsection{Atomic Force Microscopy}

Rectangular PEG hydrogels ( $5 \mathrm{~mm}$ height, $5 \mathrm{~mm}$ width, $1 \mathrm{~mm}$ thick) were made following methods described above. An atomic force microscope (MFP-3D Classic, Santa Barbara, CA) was used to measure the spatially varying elastic moduli of both the single layer and bilayer hydrogels. Briefly, force spectroscopy (forcevolume mapping) was performed in deionized water at $25^{\circ} \mathrm{C}$ in a fluid cell for the MFP-3D AFM. For the single layer hydrogels, the measurements were carried out at each node of a $64 \times 64$ grid over a $10 \mu \mathrm{m} \times 10 \mu \mathrm{m}$ area, while for the bilayer hydrogels, the measurements were carried out at each node of a $512 \times 64$ grid over an $80 \mu \mathrm{m} \times 10 \mu \mathrm{m}$ area. Bruker SNL-10-B AFM probes (Camarillo, CA) with triangular SiN cantilevers and Si tips were used. Each probe was calibrated with the thermal fluctuation method (Hutter and Bechhoefer, 1993); resulting values for the spring constant $k_{c}$ varied from $0.08 \mathrm{~N} / \mathrm{m}$ to $0.16 \mathrm{~N} / \mathrm{m}$. Moreover, each probe was inspected before and after force spectroscopy with scanning electron microscopy (SEM) to assess the half-angle $\alpha$ and check for damage or material transfer; $\alpha$ varied from $15^{\circ}$ to $23^{\circ}$ and minimal material transfer was observed. AFM force-displacement $(F-d)$ data was acquired with a peak load of $20 \mathrm{nN}$ at a displacement rate of $10 \mu \mathrm{m} / \mathrm{s}$. Force-deformation $(F-\delta)$ data were derived from the raw $F-d$ curves by 
subtracting the cantilever deflection $F / k_{c}$. To assess Young's modulus $E$ at each node, the loading portion of each $F$ - $\delta$ curve was fit to an analytical model for a rigid conical tip in contact with an elastic half-space (Sneddon, 1965), i.e., $\mathrm{F}=(2 / \pi)\left(E / 1-v^{2}\right)(\tan \alpha) \delta^{2}$, using an average value for $\alpha$ from SEM, an assumed Poisson's ratio for the hydrogel $(v=0.5)$, and $E$ as the sole fitting parameter.

\subsection{Nanoindentation}

Rectangular PEG hydrogels ( $5 \mathrm{~mm}$ height, $5 \mathrm{~mm}$ width, $1 \mathrm{~mm}$ thick) were made as described above for nanoindentation testing. A Ti-950 Triboindenter (Hysitron, Minneapolis, MN) performed micro scale indentation of single layer and bilayer hydrogels. Five samples were submerged in deionized water at $27^{\circ} \mathrm{C}$ and a fluid cell $100 \mu \mathrm{m}$ radius cono-spherical probe was pressed into the samples to a depth of $10 \mu \mathrm{m}$ at a rate of $2 \mu \mathrm{m} / \mathrm{s}$. Each sample was indented 300 times in two interlocking arrays. The first array was 20 indents wide by 8 indents tall with $100 \mu \mathrm{m}$ spacing in $\mathrm{x}$ and $200 \mu \mathrm{m}$ spacing in $\mathrm{y}$. The second array had the same spacing, but was 20 indents wide by 7 indents tall with an offset from the starting location of the first array by $50 \mu \mathrm{m}$ in $\mathrm{x}$ and $100 \mu \mathrm{m}$ in $\mathrm{y}$. To avoid effects from adjacent tests, all indents were spaced at three times the contact radius. Because the material behaved elastically the reduced modulus at each indent location was found using the maximum force and depth for each load displacement curve. The testing was performed on an extended displacement stage (Hysitron, xZ 500 Extended Displacement Stage), the load function was trapezoidal ( 5 second load, 30 second hold and 5 second unload), the Poisson ratio was assumed to be 0.5 for the samples, and the Hertz analytical method was used to evaluate the elastic modulus (Fischer-Cripps, 2002; Hertz, 1881).

\subsection{Bead Tracking and Image Processing}

Cylindrical PEG hydrogels (5 $\mathrm{mm}$ height and $5 \mathrm{~mm}$ diameter) were made following methods described above. Silicon dioxide microparticles (5 $\mu \mathrm{m}$; Sigma-Aldrich) were entrapped into each hydrogel layer at a concentration of $0.3 \%$ solids. The density of microparticles was chosen to be low enough so as not to alter the mechanical properties of the hydrogel, while enabling visualization of multiple beads in the hydrogel by a bright field microscope (Zeiss Axiovert $40 \mathrm{C}$ ). To minimize microparticle settling, the macromer solution was 
vortexed immediately before polymerization. To distinguish between the layers, PolyFlour 570

(Methacryloxyethyl Thiocarbonyl Rhodamine B; Polysciences Inc., Warrington, PA) was added to the first (i.e., bottom) layer creating a red tint. These samples were then cut in half with a razor blade, placed cut side down on a windowed compression rig, loaded in compression, and images taken at $0.5 \%$ strain increments. A MATLAB based Digital Image Correlation routine (Jones, 2013; Jones et al., 2014) was used to track the movement of the microparticles in the direction of the applied strain and extract hydrogel deformation. These deformations were then applied to a two dimensional (2D) finite element model to compute sample strain maps for each image.

\subsection{Statistics}

Statistics were performed using KaleidaGraph 4.1.3 software (Synergy Software, Reading, PA). Significant differences were established using a one-way Analysis of Variance with soak time as a factor. Comparisons between bilayer hydrogels and single layer hydrogels were performed by an unpaired, equal variance twosample t-test. P-values are provided to indicate significance. All numerical results are presented as mean (standard deviation) and graphical results are presented as mean with standard deviation as error bars $(n=3)$.

\section{Results}

\subsection{Fabrication of bilayer hydrogels}

Bilayer hydrogels were formed with covalently tethered fluorophores to enable visualization of each layer and the interface. The precursor solution for the first layer contained a red fluorophore and was polymerized. The precursor solution for the second layer contained a green fluorophore and was gently applied on top of the first layer. The time prior to exposing the second layer to light, herein referred to as soak time $\left(\mathrm{t}_{\mathrm{s}}\right)$, was varied from 0 to $20 \mathrm{~min}$. A schematic of the process is shown in Fig. 1A. The process resulted in interfaces that were visualized by the overlap of the two fluorophore channels (Fig. 1B). Four different hydrogel formulations, which are referred to as Hydrogels I-IV, with varying properties from soft to stiff were investigated to form the bilayer hydrogels. The formulation and resulting properties for each hydrogel formulation are given in Fig. 1C. The equilibrium swelling ratio varied from 18 for Hydrogel II to 10 for 
Hydrogel IV, while the mesh size varied from 42 to $23 \mathrm{~nm}$, respectively. The compressive modulus also varied from 75 to $250 \mathrm{kPa}$ for hydrogel conditions (Fig. 1C). Three bilayer hydrogels were fabricated from the different hydrogel formulations to form Bilayer III:III, Bilayer II:III, and Bilayer IV:I, where the first Roman numeral corresponds to the hydrogel formulation in the first layer and the second Roman numeral corresponds to the hydrogel formulation in the second layer (Fig. 1D). Representative photographs for each bilayer condition after reaching equilibrium swelling are shown in Fig. 1E.

\subsection{Increased time for transport leads to larger interfaces but no effect on bulk compressive modulus}

Bilayer III:III was used to investigate the effect of soak time $\left(t_{s}\right)$ on the resulting interface and macroscopic properties in the form of compressive modulus. The interface thickness increased $(p<0.0001)$ with soak time, for example from $65(14) \mu \mathrm{m}$ with a ts of $0 \mathrm{~min}$ to $230(17) \mu \mathrm{m}$ with a $\mathrm{t}_{\mathrm{s}}$ of $20 \mathrm{~min}$ (Figs. $2 \mathrm{~A}-\mathrm{C}$ ). The interface thickness was $\sim 1-4 \%$ of the total hydrogel height (Fig. 2C). The effect of soak time did not significantly affect compressive modulus of the bilayer hydrogels at low $(p=0.47)$ or high $(p=0.092)$ strains. The combination of two layers to form a bilayer hydrogel did not significantly affect ( $p=0.11-0.74$ ) the compressive modulus when compared to the single layer Hydrogel III (Fig. 2D). All bilayer hydrogels remained intact with no observable signs of damage at strains of up to $45 \%$.

\subsection{An increase in mesh size leads to larger interfaces and affects the bulk compressive modulus}

Bilayer II:III was used to investigate the effect of a larger mesh size in the first layer on the resulting interface thickness and compressive modulus. The interface thickness increased $(p<0.0001)$ with increasing soak time, for example from $130(22) \mu \mathrm{m}$ with a $\mathrm{t}_{\mathrm{s}}$ of $0 \mathrm{~min}$ to $580(5) \mu \mathrm{m}$ with a $\mathrm{t}_{\mathrm{s}}$ of $20 \mathrm{~min}$ (Fig. 3A). The overall thickness of the interface was substantially larger in this bilayer hydrogel compared to Bilayer III:III. For example, at $t_{s}=20$ min the interface thickness was 2.5-fold larger in Bilayer II:III compared to Bilayer III:III. The interfacial thickness was $\sim 2-10 \%$ of the total hydrogel height (Fig. 3C). The compressive modulus of Bilayer II:III was not significantly affected by soak time at the low $(p=0.13)$ or high $(p=0.21)$ strains with no observable trends (Fig. 3C). At low strains, the compressive modulus of the bilayer hydrogels for all soak times was either lower or not different ( $p=0.006-0.49$ ) when compared to the single layer Hydrogel II, but was lower ( $p=0.001-0.07$ ) when compared to the single layer Hydrogel III (i.e., the more highly crosslinked 
hydrogel) (Fig. 3D). At high strains, the compressive modulus of the bilayer hydrogels for all soak times was higher ( $p=0.038-0.14)$ than Hydrogel II, and remained lower ( $p=0.002-0.01)$ than Hydrogel III (Fig. 3D). None of the bilayer conditions delaminated or showed any observable signs of failure up to $45 \%$ strain.

\subsection{Smaller mesh size does not affect interface thickness, but affects the bulk compressive modulus}

Bilayer IV:I was used to investigate the effects of a smaller mesh size in the first layer on the resulting interface thickness and the compressive modulus. The mean interface thickness ranged from 140-210 $\mu \mathrm{m}$ with soak time, but there were no observable trends ( $p=0.013$ ) (Fig. 4A). The interface thickness was $\sim 3-4 \%$ of the total hydrogel height (Fig. 4C). The compressive modulus of the bilayer hydrogels was affected ( $p=$ $0.0005)$ by soak time at low strains and affected $(p=0.006)$ by soak time at the high strains, but there were no observable trends. At low strains, the compressive modulus of the bilayer hydrogels for all soak times was higher ( $p=0.002-0.27)$ than single layer Hydrogel I, but lower $(p=0.0003-0.0008)$ than single layer Hydrogel IV (Fig. 4D). At high strains, the compressive modulus of the bilayer hydrogels for all soak times was higher ( $p$ $=0.0001-0.0005)$ than Hydrogel I, but lower $(p=0.0005-0.0037)$ than Hydrogel IV. None of the bilayer conditions delaminated or showed any observable signs of failure up to $45 \%$ strain.

\subsection{Smaller mesh size inhibits diffusion of monomer into the first hydrogel layer}

When the second precursor solution is applied to the already polymerized first layer, the monomers from the precursor solution will diffuse into the first layer hydrogel. To characterize this diffusion, diffusive transport of a fluorescently labeled version of each monomer, the 8-arm PEG and the PEG crosslinker, into Hydrogel II, III, and IV was measured. A one-dimensional experimental set-up shown in Fig. 5A was used to experimentally measure concentration as a function of distance and time. The molecular weight of each fluorescently labeled molecule was similar to the corresponding monomer. To determine the diffusion coefficient for each monomer in each of the hydrogels, Fickian diffusion was assumed and the experimental data were fit to

$$
C(x, t)=C_{0} \operatorname{erfc}\left(\frac{x}{2 \sqrt{D t}}\right)
$$


where $C$ is concentration of the fluorescently labeled PEG molecule, $C_{0}$ is the concentration in the reservoir, which was held constant, $D$ is the diffusion coefficient, $x$ is distance and $t$ is time. This equation was determined by solving the simple case of diffusion with time $t$ in one dimension (taken as the $x$-axis) with boundary conditions $C(x, 0)=0$ at $x>0$ and $C(x, 0)=C_{0}$ at $x=0$. Select data are shown in Fig. 5B along with the estimated diffusion coefficients for the fluorescently labeled PEG monomers in each of three hydrogels (Fig. 5C). As expected, hydrogels with a smaller mesh size resulted in lower diffusivities for both monomers. In addition, due to its size, the smaller monomer, the PEG crosslinker, diffused to a greater extent as compared to the larger 8-arm PEG monomer. Using these diffusivities, the concentration profile of each monomer as it diffuses into the first layer, can be estimated for each bilayer condition. The initial concentration was assumed to be the concentration of the respective monomer in the precursor solution for the second hydrogel layer, where Hydrogel III had a 34 mM PEGdt and 8.1 mM PEGnor concentration, and Hydrogel I had a 21 mM PEGdt and 5.1 mM PEGnor concentration. The concentration profile of each monomer into the first layer was plotted at a time of $20 \mathrm{~min}$, corresponding to the $t_{s}=20 \mathrm{~min}$ condition, and is shown in Fig. 5D. The interface that was experimentally measured in the bilayer hydrogels for the $t_{s}=20 \mathrm{~min}$ condition (i.e., in Fig. 2C, 3C, and 4C) was superimposed on each plot. This overlay can then be used to predict, based on Fickian diffusion, the concentration of the PEG monomers within the interfacial layer. For Bilayer II:III, the concentration of PEGdt ranged from $34 \mathrm{mM}$ to $2.9 \mathrm{mM}$ and that of the PEGnor ranged from $8.1 \mathrm{mM}$ to $9 \times 10^{-8} \mathrm{mM}$. For Bilayer III:III, the concentration of PEGdt ranged from $34 \mathrm{mM}$ to $6.6 \mathrm{mM}$ and that of the PEGnor ranged from $8.1 \mathrm{mM}$

to $6 \times 10^{-3} \mathrm{mM}$. Finally, for Bilayer IV:I, the concentration of PEGdt ranged from $21 \mathrm{mM}$ to $2.4 \mathrm{mM}$ and that of the PEGnor ranged from $5.1 \mathrm{mM}$ to $0.01 \mathrm{mM}$.

\subsection{Spatial mapping of local mechanical properties reveals a gradual transition in elastic modulus at the interface of a bilayer hydrogel}

To characterize the local mechanical properties across the interface, nanoindentation and AFM were performed. When used together, nanoindentation is capable of mapping modulus over large, millimeter-sized 2D surfaces and AFM facilitates measurement of modulus variations across small, sub-millimeter regions with nanometer-scale spatial resolution. To evaluate how hydrogel composition influenced the properties of the interface, we selected Bilayer IV:I because it exhibited the largest difference in compressive modulus 
between the two layers. We also selected the $t_{s}=0$ min condition since soak time played less of a role for this hydrogel composition. With AFM, the modulus of single layer counterparts of each hydrogel, Hydrogel I and IV, was first evaluated (Fig. 6A) and the resulting values largely agree with bulk compressive modulus measurements at 10-15\% strains (Fig. 4D). In the bilayer hydrogel, AFM demonstrated a gradient in the modulus from the stiff Hydrogel IV layer to the soft Hydrogel I layer that spanned $\sim 70 \mu \mathrm{m}$ of the $80 \mu \mathrm{m}$-long scan region (Fig. 6B). Nanoindentation was also used to evaluate moduli of the individual layers and the interface region. Nanoindentation moduli, collected at low $(\sim 4 \%)$ strains when tested using a large radius cono-spherical probe produced correspondingly lower modulus values for the hydrogels, as compared to values from AFM and unconfined compression (Fig. 6C). Nanoindentation also demonstrated a graded transition of indentation modulus from the stiff to the soft layer (Fig. 6C).

\subsection{Strain transfer depends on the local mechanical properties of each layer.}

Finite strains within Bilayer IV:I were determined by bead tracking and digital image correlation techniques under compressive loading. Bilayer IV:I was chosen because it exhibited the largest difference in compressive modulus between the two layers. Spherical silicon dioxide beads ( $5 \mu \mathrm{m}$ in diameter), encapsulated within hydrogels, are much larger than the mesh size of the hydrogels and therefore their movement can be correlated to the local strain of the hydrogel. Digital image correlation methods and a 2D model were used to calculate finite strains across the bilayer hydrogel. Under a 15\% strain applied to the bilayer hydrogel, a color map of the strain distribution within the hydrogel was generated (Fig. 7A). The soft layer experienced $\sim 20 \%$ strain while the stiff layer experienced $\sim 10 \%$ strain. The strains were plotted as a function of position for increasing bulk strains (Fig. 7B). Across the interface, the strain levels progressively decreased from the soft to the stiff layer. The variation in the strain from the soft to stiff layers occurred over a relatively large length scale of $\sim 1 \mathrm{~mm}$.

\section{Discussion}

The overall objectives for this study were to characterize how the interface forms via its thickness and mechanical properties, identify its impact on the overall hydrogel properties, and provide new insights into 
how to control the interface when multi-layer hydrogels are formed from sequential polymerization of monomers in a step-and-repeat process. Several factors controlled the thickness of the interface and included soak time (i.e., time before the second layer is polymerized) and the mesh size of the first layer. The interface thickness ranged from $\sim 70$ to $600 \mu \mathrm{m}$ depending on these factors, but did not influence the bulk modulus measurements. We further showed that the interface results in a gradient in mechanical properties when bilayer hydrogels are formed from soft and stiff layers and this translated to a gradient in strain transfer across the interface under a gross applied strain. Overall, the study shows that a mechanically robust interface forms, but whose thickness can be controlled through the choice of processing conditions.

In the step-and-repeat process, the second hydrogel precursor solution is deposited on top of a crosslinked hydrogel immediately after it is polymerized. There are two potential factors that can influence the formation of the interface and its thickness: (1) transport by diffusion of monomers into the first hydrogel layer (Amsden, 1998a, 1998b; Hagel et al., 2013; Lustig and Peppas, 1988) and (2) transport by convection of monomers due to changes in swelling of the first hydrogel layer (Brannon-Peppas and Peppas, 1991; Kim et al., 1992; Martens and Anseth, 2000). Monomer transport by diffusion will occur due to a concentration gradient, but will depend on the relative size of the monomers in the second layer to the mesh size of the hydrogel in the first layer. Transport by convection occurs when the solvent concentration in the precursor solution differs from that at the equilibrium state of the swollen hydrogel, leading to rapid changes in swelling in the first hydrogel layer when the second layer is applied. Both transport mechanisms likely contribute to the formation of the interface in the bilayer PEG hydrogels. The largest interface was formed when the first layer was fabricated from Hydrogel II. The relatively large interface of $\sim 130 \mu \mathrm{m}$ at the initial time point suggests transport by convection due to a rapid initial swelling response. Indeed, this hydrogel swelled 3.9x its initial mass when placed in an aqueous solvent, which can be seen in Fig. 1E. With longer soak times, the interface continued to grow. The relatively large mesh size of Hydrogel II led to the highest diffusivity for the PEG crosslinker and the 8-arm PEGnor monomer, enabling both monomers to diffuse into the first layer. Therefore, it is reasonable to conclude that transport by diffusion is playing a dominant role in the observed increased interface thickness with soak time. When a more tightly crosslinked hydrogel (i.e., Hydrogel III) was used to fabricate the first layer, the overall interface thickness followed a similar trend, but 
was smaller (e.g., initial interface thickness was $\sim 70 \mu \mathrm{m}$ ). This observation is supported by a lower initial swelling response, shown in Fig. 1E where Hydrogel III swelled 2.5x its initial mass when placed in an aqueous solvent, and also had a lower diffusivity compared to Hydrogel II. Taken together, the thickness of the interface is dependent on both convective and diffusive transport.

Regardless of the transport mechanism, the transported monomers will subsequently be able to react with free norbornenes and/or thiols within the first layer and/or with each other to form a semi-interpenetrating network. Non-idealities that result during network formation will leave free reactive groups in the first layer enabling covalent bonds to form between the two layers (Dušek and Dušková-Smrčková, 2000; Elliott and Bowman, 2002; Elliott et al., 2004, 2003; Metters and Hubbell, 2005). Both the 8-arm PEGnor monomer and the PEGdt crosslinker were found to diffuse into the first layer, but the concentration of each monomer via diffusive transport drops quickly and more so for the larger 8-arm PEGnor monomer. When the concentration of the 8-arm PEGnor drops below $\sim 2 \mathrm{mM}$, a network is unable to form. Thus for diffusive transport, it can be reasoned that an interface forms due to covalent bonds and to a lesser extent by the formation of a semi-interpenetrating network. However, when convective transport dominates, the interface that forms is likely a combination of covalent bonds and semi-interpenetrating networks.

Interestingly, interface thickness did not vary with soak time when the first layer hydrogel was fabricated from Hydrogel IV, the stiffest hydrogel used in this study. However, the interface thickness at the initial time point was relatively large at $\sim 140 \mu \mathrm{m}$. It was observed that Hydrogel IV, like Hydrogel II, undergoes significant swelling ( $\sim 3.2 \mathrm{x}$ its initial mass) after polymerization to reach equilibrium, which can be seen in Fig. 1E. Thus, it is not surprising that the interface thickness at the initial time point was large for this hydrogel formulation, similar to the case with Hydrogel II. The lower diffusivity of the PEGdt and PEGnor, however, led to reduced diffusion into the hydrogel over the 20 minutes of soak time and as a result no further increase in the interface thickness was observed with longer soak times.

Taken together, these observations support the idea that for these PEG hydrogels, a rapid swelling response leads to the formation of an initial interface, which arises due to convective transport of the monomers into 
the first layer hydrogel. The initial thickness of the interface depends on how far the solvent concentration in the precursor solution is from the equilibrium-swollen volume of the hydrogel. The farther from the equilibrium-swollen volume, the larger the resulting initial interface will be. However, with longer soak times, the interface grows predominantly due to diffusion of the monomers into the previously polymerized hydrogel layer. By controlling the relative size of the monomers to that of the mesh size of the first layer, it is possible to further control the thickness of the interface and even prevent it from growing, at least at short soak times.

Mechanically, the bilayer hydrogels formed from sequential polymerization of thiol-ene photo-clickable monomers led to robust hydrogels that supported large compressive strains (up to 45\%) with no observable signs of failure. The robust interface that links the two layers is attributed to a combination of covalent bonds and entanglements that form as the monomers are transported into the first layer and subsequently polymerized. The overall compressive modulus of the bilayer hydrogels was dependent on the relative modulus of the two layers, but did not appear to depend strongly on the interface thickness. When the layers were similar in crosslink density, the overall compressive modulus of the bilayer hydrogels was similar to the single layer hydrogels. When the layers had different moduli, the overall compressive modulus mirrored that of the soft layer at low strains, but at high strains exhibited a modulus that was in between the modulus of each layer. These observations follow composite theory of materials, which also predicts that a relatively thin interface ( $\sim 10 \%$ or less of the total height) would not contribute appreciably to the overall compressive modulus (Chawla, 2012; Herakovich, 1997; Kinneberg et al., 2015).

The interfacial characteristics between adjacent layers within multi-layer hydrogels are critical to the successful distribution of strains, and prevention of interface failure, under compressive loading. From nanoand micro-mechanical measurements using both AFM and nanoindentation, respectively, we observed a gradient in mechanical properties across the interface of the Bilayer IV:I hydrogel. This observation indicates that after the first layer is polymerized, a gradient in crosslink density forms at its surface, which is consistent with the observed gradient in the fluorescence. We confirmed in a separate experiment that in single layer hydrogels a gradient appears at the free surface of the hydrogel due to swelling (DuPont Jr. et al., 2010). In 
addition, the temporal swelling behavior of hydrogels (Bell and Peppas, 1996; Brannon-Peppas and Peppas, 1991; Khare and Peppas, 1995; Martens and Anseth, 2000) will also contribute to the formation of a gradient. However, it is possible that an inverse gradient could form as monomers from the second layer are transported into the first layer and subsequently react, which will increase the local crosslink density. The latter appears to have less of an affect since a gradient from stiff to soft is observed. Overall, the combination led to a smooth, gradual transition in the modulus across the stiff-soft hydrogel interface.

The transition of mechanical properties across the interface of the Bilayer IV:I hydrogel was evaluated using a sharp probe via AFM, to evaluate changes in mechanical properties with nanometer spatial resolution. The mechanical interface between the stiff and soft layers in the bilayer hydrogel varied over a distance of approximately $70 \mu \mathrm{m}$, which is substantially smaller, by $\sim 2$-fold, than the interface thickness that was measured using confocal microscopy. This observation points to potential differences between the variation in chemistry and mechanical properties across the interface between two dissimilar hydrogels. While the PEG crosslinker molecules and to a lesser degree the 8-arm PEGnor monomers are transported farther into and react with the first layer, it is likely that not all contribute significantly to the mechanical properties. Thus, the interface thickness may be distinctly different with respect to chemistry and elastic modulus.

While AFM using a sharp probe is highly sensitive to mechanical property changes in a heterogeneous material, nanoindentation with a large sphere is better capable of accurately determining elastic moduli in hydrogels. Strains under sharp AFM probes are constant and related to the probe half-angle, yet are difficult to quantify exactly (Atkins and Tabor, 1965; Johnson, 1970). As hydrogels exhibit a nonlinear relationship between stress and strain (Anseth et al., 1996), moduli determined from AFM without knowing the corresponding strain is challenging. Comparably, probing with a large sphere enables facile determination of strains and maintains criteria needed for Hertzian contact mechanics (i.e., when small strains are maintained), and so permits evaluation of elastic moduli at known strain values. In this study, nanoindentation using a $100 \mu \mathrm{m}$ cono-spherical probe produced relatively small, $\sim 4 \%$ strain in the hydrogel volume compressed by the indenter probe. Moreover, commercial nanoindenter systems enable mechanical property (e.g., elastic modulus) mapping over large (centimeter) regions in comparison to limited range with 
AFM (max of $\sim 200 \mu \mathrm{m}$ in $\mathrm{x}$ - and y-direction). Evaluation of the Bilayer IV:I hydrogel using nanoindentation revealed a gradual modulus gradient, over a distance of $\sim 300 \mu \mathrm{m}$, between the two hydrogel layers with a transition region from $>150 \mathrm{kPa}$, in Hydrogel IV, to $\sim 60 \mathrm{kPa}$, in Hydrogel I. These moduli compare well with properties observed at comparable strains in unconfined compression testing of single layer hydrogels in this study. The wide distance indicating the interface of the bilayer hydrogel, as compared to the $70 \mu \mathrm{m}$ mechanical interface observed using AFM, results from the large contact radius $(\sim 32.4 \mu \mathrm{m})$ of the conospherical nanoindenter probe, where the material within roughly $3-3.5$ times that of the contact radius contributes to the measured elastic modulus. Thus the mechanical interface revealed by nanoindentation is wider than that of AFM, where the latter possesses better spatial resolution by using a very small $(\sim 10 \mathrm{~nm})$ sharp probe. Nonetheless, nanoindentation using a large probe is able to map the modulus gradient that exists between the two dissimilar hydrogels and provide more accurate modulus values. This gradient was also mirrored in the local strain measurements as observed by strain measurements in bead tracking experiments. The length-scale over which strain varied across the bilayer interface was even greater $(\sim 1000$ $\mu \mathrm{m}$ in length) than the modulus gradient observed using nanoindentation. We attribute this finding to the experimental methods, where relatively large unconfined compressive strains (i.e., 15\%) were applied to the entire hydrogel construct. As a result of these large strains, deformation of the hydrogel leads to lateral expansion (Kinneberg et al., 2015), and therefore extends the strains over a much larger distance. Importantly, the broad strain field observed indicates that the graded interface between the two dissimilar hydrogels enables effective strain transfer between stiff and soft layers. Moreover, the strain field is distributed far from the interface in the bilayer hydrogel and does not include a sharp increase at the interface of the two materials, which would indicate potential for interfacial failure.

There are several limitations that are important to note. Our findings point towards diffusion and convection as playing an important role in the formation of the interface of the bilayer hydrogels. It was, however, not possible to decouple these factors experimentally and therefore mathematical models may be necessary to differentiate them and identify their contribution to the formation of the interface. Another limitation of this study is the lack of nanomechanical evaluation of the other bilayer conditions. Due to the similarity in mechanics of the layers in Bilayer III:III and II:III the interface is difficult to probe, and the much larger 
interfaces formed at longer soak times would be challenging to capture by the narrow grids afforded by AFM. However, understanding the properties of such interfaces formed when the first layer is composed of larger mesh sizes like that of Hydrogel II or III would be paramount in further identifying the effects of swelling and diffusion on interface mechanics. Also as discussed, due to the non-linear relationship between stress and strain exhibited by hydrogels (Kinneberg et al., 2015), AFM likely overestimated the moduli of Hydrogels IV and I. Nanoindentation of soft materials like hydrogels requires large probes (i.e., due to limited ability of commercial nanoindenters to detect low force levels), and so lacks the spatial resolution of sharp AFM probes to directly evaluate modulus gradients. Yet nanoindentation can readily evaluate a functional (e.g., modulus) gradient across the bilayer interface in large millimeter-sized arrays and provides a realistic measure of elastic modulus of each hydrogel layer and the intermediate interfacial region. While each approach has limitations, we conclude that the combination of techniques used herein enables a comprehensive understanding of small length scale $(<100 \mu \mathrm{m})$ interfacial regions at high spatial resolution (i.e., using AFM), moduli of single hydrogels and modulus gradients at interfaces (i.e., using nanoindentation) and behavior (via strain mapping) spanning large, millimeter-sized regions.

\section{Conclusions}

In this study, we characterized the interface that forms between two layers of a bilayer PEG hydrogel that is fabricated by a step-and-repeat process of photo-polymerized PEG monomers. We identified that the interface forms as a result of two phenomena: (a) convective transport of monomers, which occurs rapidly due to changes in swelling in the first layer when the second layer is applied and (b) diffusive transport of monomers, which occurs over longer time scales, and is dependent on the relative size of the monomers to the mesh size of the first layer. It is important to note that these phenomena are not specific to PEG hydrogels and therefore extend to any bilayer hydrogel that is formed by a step-and-repeat process. Mechanically, a robust interface forms as evident by no signs of failure (up to the strains tested) and the compressive modulus of the bilayer hydrogels mirrored that of the layers and not the interface. Nanomechanical analysis confirmed that a gradient in modulus forms across the interface and subsequently leads to a gradual transfer of strain across the entire bilayer hydrogel in unconfined compression. Overall, this work provides new 
insight into the mechanisms that control the formation of an interface and the resulting interfacial properties when multiple layers are sequentially polymerized. The ability to control the interface between sequentially polymerized hydrogels is important to interfacial tissue engineering applications where multi-layer hydrogels are employed.

\section{Acknowledgments}

Research reported in this publication was partially supported by the NSF CAREER Award DMR \#0847390 (A.H.A., S.J.B.) and NSF CAREER Award CBET \#1055989 (J.W., V.L.F.), NIH Pharmaceutical Biotechnology Training fellowship (A.H.A.), GAANN Functional Material Grant (A.H.A.), and NIST Measurement Science \& Engineering Fellowship (A.H.A.). The authors acknowledge the NSF Major Research Instrumentation Award (NSF CBET\#1338154) and the University of Colorado, Boulder CO, USA, for funding the combined Raman spectroscopy - nanoindenter system used in this work. Specific commercial equipment, instruments, and materials that are identified in this report are listed in order to adequately describe the experimental procedure and are not intended to imply endorsement or recommendation by the National Institute of Standards and Technology (NIST). 


\section{References}

Akalp, U., Chu, S., Skaalure, S.C., Bryant, S.J., Doostan, A., Vernerey, F.J., 2015. Determination of the polymersolvent interaction parameter for PEG hydrogels in water: Application of a self learning algorithm. Polymer (Guildf). 66, 135-147. doi:10.1016/j.polymer.2015.04.030

Amsden, B., 1998a. Solute Diffusion within Hydrogels. Mechanisms and Models. Macromolecules 31, 83828395. doi:10.1021/ma980765f

Amsden, B., 1998b. Solute diffusion in hydrogels. Polym. Gels Networks 6, 13-43. doi:10.1016/S09667822(97)00012-9

Anseth, K.S., Bowman, C.N., Brannon-Peppas, L., 1996. Mechanical properties of hydrogels and their experimental determination. Biomaterials 17, 1647-1657. doi:10.1016/0142-9612(96)87644-7

Atkins, A.G., Tabor, D., 1965. Plastic indentation in metals with cones. J. Mech. Phys. Solids 13, 149-164. doi:10.1016/0022-5096(65)90018-9

Bell, C.L., Peppas, N.A., 1996. Water, solute and protein diffusion in physiologically responsive hydrogels of poly(methacrylic acid-g-ethylene glycol). Biomaterials 17, 1203-1218. doi:10.1016/01429612(96)84941-6

Brannon-Peppas, L., Peppas, N.A., 1991. Equilibrium swelling behavior of pH-sensitive hydrogels. Chem. Eng. Sci. 46, 715-722. doi:10.1016/0009-2509(91)80177-Z

Campbell, S.E., Ferguson, V.L., Hurley, D.C., 2012. Nanomechanical mapping of the osteochondral interface with contact resonance force microscopy and nanoindentation. Acta Biomater. 8, 4389-4396. doi:10.1016/j.actbio.2012.07.042

Canal, T., Peppas, N.A., 1989. Correlation between mesh size and equilibrium degree of swelling of polymeric networks. J. Biomed. Mater. Res. 23, 1183-1193. doi:10.1002/jbm.820231007

Chawla, K.K., 2012. Composite Materials. Springer New York, New York, NY. doi:10.1007/978-0-387-74365-3

DuPont Jr., S.J., Cates, R.S., Stroot, P.G., Toomey, R., 2010. Swelling-induced instabilities in microscale, surfaceconfined poly(N-isopropylacryamide) hydrogels. Soft Matter 6, 3876. doi:10.1039/c0sm00021c

Dušek, K., Dušková-Smrčková, M., 2000. Network structure formation during crosslinking of organic coating systems. Prog. Polym. Sci. 25, 1215-1260. doi:10.1016/S0079-6700(00)00028-9 
Elliott, J.E., Bowman, C.N., 2002. Effect of Primary Cyclization on Free Radical Polymerization Kinetics: Modeling Approach. Macromolecules 35, 7125-7131. doi:10.1021/ma012001x

Elliott, J.E., Macdonald, M., Nie, J., Bowman, C.N., 2004. Structure and swelling of poly(acrylic acid) hydrogels: effect of $\mathrm{pH}$, ionic strength, and dilution on the crosslinked polymer structure. Polymer (Guildf). 45, 1503-1510. doi:10.1016/j.polymer.2003.12.040

Elliott, J.E., Nie, J., Bowman, C.N., 2003. The effect of primary cyclization on free radical polymerization kinetics: experimental characterization. Polymer (Guildf). 44, 327-332. doi:10.1016/S00323861(02)00734-6

Erdogan, F., 1995. Fracture Mechanics of Functionally Graded Materials. MRS Bull. 20, 43-44. doi:10.1557/S0883769400048934

Fairbanks, B.D., Schwartz, M.P., Halevi, A.E., Nuttelman, C.R., Bowman, C.N., Anseth, K.S., 2009. A Versatile Synthetic Extracellular Matrix Mimic via Thiol-Norbornene Photopolymerization. Adv. Mater. 21, 50055010. doi:10.1002/adma.200901808

Fischer-Cripps, A.C., 2002. Nanoindentation, Mechanical Engineering Series. Springer New York, New York, NY. doi:10.1007/978-0-387-22462-6

Fischer, M., Vahdatzadeh, M., Konradi, R., Friedrichs, J., Maitz, M.F., Freudenberg, U., Werner, C., 2015. Multilayer hydrogel coatings to combine hemocompatibility and antimicrobial activity. Biomaterials 56, 198-205. doi:10.1016/j.biomaterials.2015.03.056

Flory, P.J., 1953. Principles of Polymer Chemistry, 1st ed. Cornell University Press.

Flory, P.J., Erman, B., 1982. Theory of elasticity of polymer networks. 3. Macromolecules 15, 800-806. doi:10.1021/ma00231a022

Fuoco, C., Salvatori, M., Biondo, A., Shapira-Schweitzer, K., Santoleri, S., Antonini, S., Bernardini, S., Tedesco, F.S., Cannata, S., Seliktar, D., Cossu, G., Gargioli, C., 2012. Injectable polyethylene glycol-fibrinogen hydrogel adjuvant improves survival and differentiation of transplanted mesoangioblasts in acute and chronic skeletal-muscle degeneration. Skelet. Muscle 2, 24. doi:10.1186/2044-5040-2-24

Hagel, V., Haraszti, T., Boehm, H., 2013. Diffusion and interaction in PEG-DA hydrogels. Biointerphases 8, 36. doi:10.1186/1559-4106-8-36

Herakovich, C.T., 1997. Mechanics of Fibrous Composites. Wiley, New York, NY. 
Hertz, H., 1881. On the contact of elastic solids. J. für die reine und Angew. Math. 110, 156-171.

Holmes, M.H., Mow, V.C., 1990. The nonlinear characteristics of soft gels and hydrated connective tissues in ultrafiltration. J. Biomech. 23, 1145-1156. doi:10.1016/0021-9290(90)90007-P

Hoyle, C.E., Bowman, C.N., 2010. Thiol-Ene Click Chemistry. Angew. Chemie Int. Ed. 49, 1540-1573. doi:10.1002/anie.200903924

Hutter, J.L., Bechhoefer, J., 1993. Calibration of atomic-force microscope tips. Rev. Sci. Instrum. 64, 1868. doi:10.1063/1.1143970

Hwang, Y., Sangaj, N., Varghese, S., 2010. Interconnected Macroporous Poly(Ethylene Glycol) Cryogels as a Cell Scaffold for Cartilage Tissue Engineering. Tissue Eng. Part A 16, 3033-3041. doi:10.1089/ten.tea.2010.0045

Johnson, K.L., 1970. The correlation of indentation experiments. J. Mech. Phys. Solids 18, 115-126. doi:10.1016/0022-5096(70)90029-3

Jones, E.M.C., 2013. Improved digital image correlation.

Jones, E.M.C., Silberstein, M.N., White, S.R., Sottos, N.R., 2014. In Situ Measurements of Strains in Composite Battery Electrodes during Electrochemical Cycling. Exp. Mech. 54, 971-985. doi:10.1007/s11340-014$9873-3$

Kandel, R.A., Grynpas, M., Pilliar, R., Lee, J., Wang, J., Waldman, S., Zalzal, P., Hurtig, M., 2006. Repair of osteochondral defects with biphasic cartilage-calcium polyphosphate constructs in a Sheep model. Biomaterials 27, 4120-4131. doi:10.1016/j.biomaterials.2006.03.005

Ker, R., 2007. Mechanics of tendon, from an engineering perspective. Int. J. Fatigue 29, 1001-1009. doi:10.1016/j.ijfatigue.2006.09.020

Khare, A.R., Peppas, N.A., 1995. Swelling/deswelling of anionic copolymer gels. Biomaterials 16, 559-567. doi:10.1016/0142-9612(95)91130-Q

Kim, S.W., Bae, Y.H., Okano, T., 1992. Hydrogels: Swelling, Drug Loading, and Release. Pharm. Res. 09, 283290. doi:10.1023/A:1015887213431

Kinneberg, K.R.C., Nelson, A., Stender, M.E., Aziz, A.H., Mozdzen, L.C., Harley, B.A.C., Bryant, S.J., Ferguson, V.L., 2015. Reinforcement of Mono- and Bi-layer Poly(Ethylene Glycol) Hydrogels with a Fibrous Collagen Scaffold. Ann. Biomed. Eng. 43, 2618-2629. doi:10.1007/s10439-015-1337-0 
Lin, H.-Y., Chen, S.-H., Chang, S.-H., Huang, S.-T., 2015. Tri-layered chitosan scaffold as a potential skin substitute. J. Biomater. Sci. Polym. Ed. 26, 855-867. doi:10.1080/09205063.2015.1061350

Lin, L., Zhu, J., Kottke-Marchant, K., Marchant, R.E., 2014. Biomimetic-Engineered Poly (Ethylene Glycol) Hydrogel for Smooth Muscle Cell Migration. Tissue Eng. Part A 20, 140109062102007. doi:10.1089/ten.tea.2013.0050

Lustig, S.R., Peppas, N.A., 1988. Solute diffusion in swollen membranes. IX. Scaling laws for solute diffusion in gels. J. Appl. Polym. Sci. 36, 735-747. doi:10.1002/app.1988.070360401

Martens, P., Anseth, K.., 2000. Characterization of hydrogels formed from acrylate modified poly(vinyl alcohol) macromers. Polymer (Guildf). 41, 7715-7722. doi:10.1016/S0032-3861(00)00123-3

Merrill, E.W., Dennison, K.A., Sung, C., 1993. Partitioning and diffusion of solutes in hydrogels of poly(ethylene oxide). Biomaterials 14, 1117-1126. doi:10.1016/0142-9612(93)90154-T

Metters, A., Hubbell, J., 2005. Network Formation and Degradation Behavior of Hydrogels Formed by MichaelType Addition Reactions. Biomacromolecules 6, 290-301. doi:10.1021/bm049607o

Nguyen, L.H., Kudva, A.K., Saxena, N.S., Roy, K., 2011. Engineering articular cartilage with spatially-varying matrix composition and mechanical properties from a single stem cell population using a multi-layered hydrogel. Biomaterials 32, 6946-6952. doi:10.1016/j.biomaterials.2011.06.014

Nguyen, Q.T., Hwang, Y., Chen, A.C., Varghese, S., Sah, R.L., 2012. Cartilage-like mechanical properties of poly (ethylene glycol)-diacrylate hydrogels. Biomaterials 33, 6682-6690. doi:10.1016/j.biomaterials.2012.06.005

Nicodemus, G.D., Bryant, S.J., 2008. Cell Encapsulation in Biodegradable Hydrogels for Tissue Engineering Applications. Tissue Eng. Part B Rev. 14, 149-165. doi:10.1089/ten.teb.2007.0332

Paxton, J.Z., Donnelly, K., Keatch, R.P., Baar, K., 2009. Engineering the Bone-Ligament Interface Using Polyethylene Glycol Diacrylate Incorporated with Hydroxyapatite. Tissue Eng. Part A 15, 1201-1209. doi:10.1089/ten.tea.2008.0105

Peppas, N.A., 1986. Hydrogels in medicine and pharmacy, in: Volume 1: Fundamentals. CRC Press, Boca Raton, pp. 27-56.

Roberts, J.J., Bryant, S.J., 2013. Comparison of photopolymerizable thiol-ene PEG and acrylate-based PEG hydrogels for cartilage development. Biomaterials 34, 9969-9979. 
doi:10.1016/j.biomaterials.2013.09.020

Sherwood, J.K., Riley, S.L., Palazzolo, R., Brown, S.C., Monkhouse, D.C., Coates, M., Griffith, L.G., Landeen, L.K., Ratcliffe, A., 2002. A three-dimensional osteochondral composite scaffold for articular cartilage repair. Biomaterials 23, 4739-4751. doi:10.1016/S0142-9612(02)00223-5

Shinohara, S., Kihara, T., Sakai, S., Matsusaki, M., Akashi, M., Taya, M., Miyake, J., 2013. Fabrication of in vitro three-dimensional multilayered blood vessel model using human endothelial and smooth muscle cells and high-strength PEG hydrogel. J. Biosci. Bioeng. 116, 231-234. doi:10.1016/j.jbiosc.2013.02.013

Sneddon, I.N., 1965. The relation between load and penetration in the axisymmetric boussinesq problem for a punch of arbitrary profile. Int. J. Eng. Sci. 3, 47-57. doi:10.1016/0020-7225(65)90019-4

Steinmetz, N.J., Aisenbrey, E.A., Westbrook, K.K., Qi, H.J., Bryant, S.J., 2015. Mechanical loading regulates human MSC differentiation in a multi-layer hydrogel for osteochondral tissue engineering. Acta Biomater. 21, 142-153. doi:10.1016/j.actbio.2015.04.015

Tibbitt, M.W., Anseth, K.S., 2009. Hydrogels as extracellular matrix mimics for 3D cell culture. Biotechnol. Bioeng. 103, 655-663. doi:10.1002/bit.22361 


\section{Figure Captions}

Figure 1. (A) Schematic depicting the formation of bilayer hydrogels by sequential photopolymerization. Bilayer hydrogels were fabricated in a sequential fashion with the first layer (red) polymerized to completion, followed by introducing a second layer (green) that is deposited for a prescribed period of time (soak time, $\mathrm{t}_{\mathrm{s}}$ ) to allow for transport of monomers prior to second polymerization. (B) Representative confocal microscopy image of the bilayer hydrogel with a bottom layer from the 25\% (w/w) PEGnor formulation (Hydrogel IV) (red) and a top layer from the $10 \%(\mathrm{w} / \mathrm{w})$ PEGnor formulation, where the interface extends across the two layers leading to a yellow band; scale bar $=200 \mu \mathrm{m}$. (C) Four hydrogel formulations are investigated in this study that were made from 10, 15 or 25\% (w/w) PEGnor to form Hydrogel I, II, III and IV respectively, and their properties are shown. (D) Three bilayer conditions (height $=5 \mathrm{~mm}$, diameter $=5 \mathrm{~mm}$ ) prepared from different hydrogel formations are investigated in this study and denoted by roman numerals. (E) Representative photographs of the cylindrical bilayer hydrogels for Bilayer III:III, Bilayer II:III, and Bilayer IV:I from left to right; scale bar $=5 \mathrm{~mm}$. *the concentration of PEGnor in the precursor solution prior to polymerization.

Figure 2. (A) Representative confocal microscopy images of Bilayer III:III, fabricated with a bottom layer from 15\% (w/w) PEGnor and $\mathrm{PEG}_{1 \mathrm{k}} \mathrm{dt}$ (red), a top layer from 15\% (w/w) PEGnor and $\mathrm{PEG}_{1 \mathrm{k}} \mathrm{dt}$ (green), and the interface (yellow); scale bar $=200 \mu \mathrm{m}$. (B) A representative plot of intensity for green and red fluorescence across the interface for Bilayer III:III. (C) Interface thickness and average percent height of the interface relative to the total height of the hydrogel as a function of soak time ( $\mathrm{t}_{\mathrm{s}}$ ) for Bilayer III:III. (D) Compressive modulus at low (10-15\%) and high (40-45\%) strains of the Bilayer III:III hydrogel and single layer Hydrogel III. Data are presented as mean $(n=3)$ with standard deviation as error bars; $p$-values in panel D denote significance from single layer hydrogel.

Figure 3. (A) Representative confocal microscopy images of Bilayer II:III, fabricated with a bottom layer from 15\% (w/w) PEGnor and PEG $_{3.4 k}$ dt (red), a top layer from 15\% (w/w) PEGnor (green) and PEG $_{1 k} d t$, and the interface (yellow); scale bar $=200 \mu \mathrm{m}$. (B) A representative plot of intensity for green and red fluorescence 
across the interface for Bilayer II:III. (C) Interface thickness and average percent height of the interface relative to the total height as a function of soak time ( $t_{s}$ ) for Bilayer II:III. (D) Compressive modulus at low (10-15\%) and high (40-45\%) strains of the Bilayer II:III and single layer Hydrogel II and Hydrogel III. Data are presented as mean ( $n=3)$ with standard deviation as error bars; p-values in panel D denote significance from single layer hydrogels; the first value from the left compares to Hydrogel II and the second value compares to Hydrogel III.

Figure 4. (A) Representative confocal microscopy images of Bilayer IV:I, fabricated with a bottom layer from 25\% (w/w) PEGnor and PEG $\mathrm{P}_{\mathrm{k}} \mathrm{dt}$ (red), a top layer from 10\% (w/w) PEGnor and $\mathrm{PEG}_{1 \mathrm{k}} \mathrm{dt}$ (green), and the interface (yellow); scale bar $=200 \mu \mathrm{m}$. (B) A representative plot of intensity for green and red fluorescence across the interface. (C) Interface thickness and average percent height of the interface relative to the total height as a function of soak time ( $\mathrm{t}_{\mathrm{s}}$ ) Bilayer IV:I. (D) Compressive modulus at low (10-15\%) and high (4045\%) strains of the Bilayer IV:I and single layer Hydrogel I and Hydrogel IV. Data are presented as mean ( $\mathrm{n}=$ 3) with standard deviation as error bars; p-values in panel D denote significance from single layer hydrogels; the first value from the left compares to Hydrogel I and the second value compares to Hydrogel IV.

Figure 5. (A) Schematic of the experimental setup for $1 D$ diffusion for each monomer (i.e., a methoxy-PEGfluorophore representing the $\mathrm{PEG}_{1 \mathrm{k}} \mathrm{dt}$ crosslinker or a fluorescently labeled 8-arm PEG representing the PEGnor monomer) each of the hydrogels used in the first layer of the bilayer hydrogels. Hydrogels were sandwiched between two glass slides and a silicone mold (gray) with reservoirs on each end. Green represents the fluorescently labeled 8-arm PEG or fluorescently labeled PEG crosslinker in solution in the reservoir and diffusing into the hydrogel. (B) Representative plots for the 1D concentration profile of fluorescently labeled PEG monomers as a function of distance into a hydrogel. Data for Hydrogel II is shown with the fluorescently labeled PEG crosslinker representing PEGdt at the 30 hour timepoint and with the fluorescently labeled 8arm PEG representing the PEGnor monomer at the 39 hour timepoint. Dotted line represents experimentally determined concentration profiles. Solid line represents the accompanying fit to the solution of Fick's second law in 1D, which was used to determine the diffusion coefficient for each hydrogel formulation and shown in (C) with mean and (standard deviation). (D) The predicted concentration 
profiles at $t_{s}=20$ minutes for each hydrogel. Dashed line represents the concentration of PEGdt as a function of distance and the dashed and dotted line represents that of the PEGnor monomer. Superimposed in gray is the experimentally determined interface as measured by confocal microscopy with a dot representing the intersection of the edge of the interface and the concentration of PEGdt due to transport by diffusion, and a square representing the intersection of the edge of the interface and the concentration of PEGnor due to transport by diffusion.

Figure 6. (A) A representative AFM image of a $64 \times 64$ grid over a $10 \mu \mathrm{m} \times 10 \mu \mathrm{m}$ area, showing the elastic moduli of single layer Hydrogel I and Hydrogel IV, respectively; accompanying force - deformation (F- $\delta$ ) data and mean elastic moduli with (standard deviation) are included. (B) Representative force - deformation (F- $\delta$ ) data, elastic moduli as a function of $\mathrm{X}$ - position, and an AFM image of a $512 \times 64$ grid over an $80 \mathrm{~mm} \times 10 \mathrm{~mm}$ area across the interface of a Bilayer IV:I hydrogel, moving from Hydrogel IV to Hydrogel I from left to right, respectively. (C) Elastic moduli (solid line) with standard deviation (dotted line) as a function of X - position across the interface of a Bilayer IV:I hydrogel, moving from Hydrogel IV to Hydrogel I from left to right respectively, as calculated from nanoindentation using the Hertz analytical method and an assumed Poisson's ratio of 0.5 .

Figure 7. (A) Representative color map of the strain distribution within Bilayer IV:I superimposed on a crosssectional image of the hydrogel, from left to right as Hydrogel IV to Hydrogel I respectively, under an applied 15\% strain. (B) Strains plotted as a function of X-position, transitioning from Hydrogel IV into Hydrogel I from left to right respectively, for increasing bulk strains. 


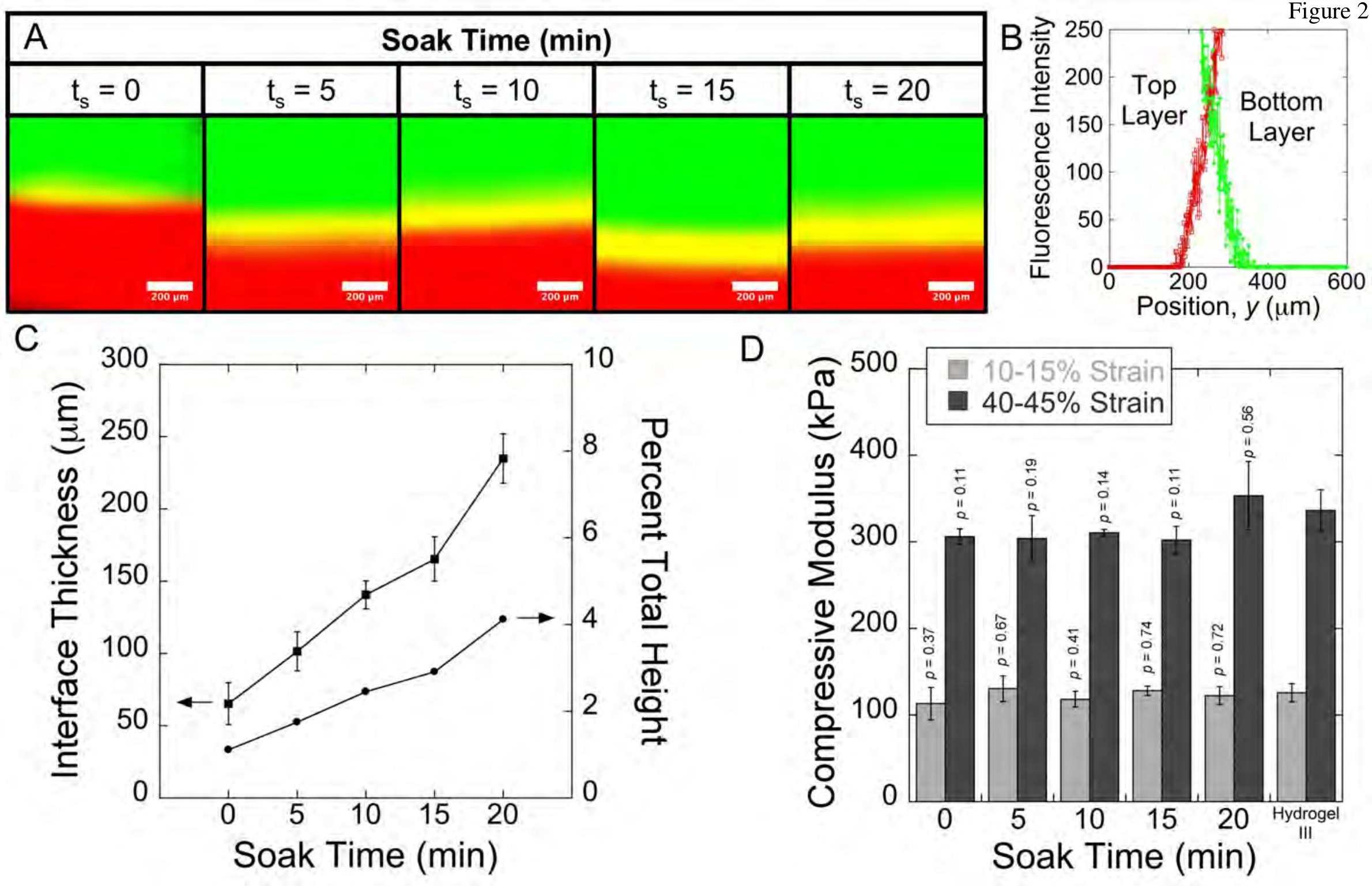




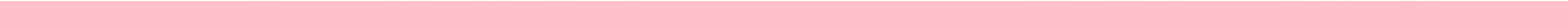




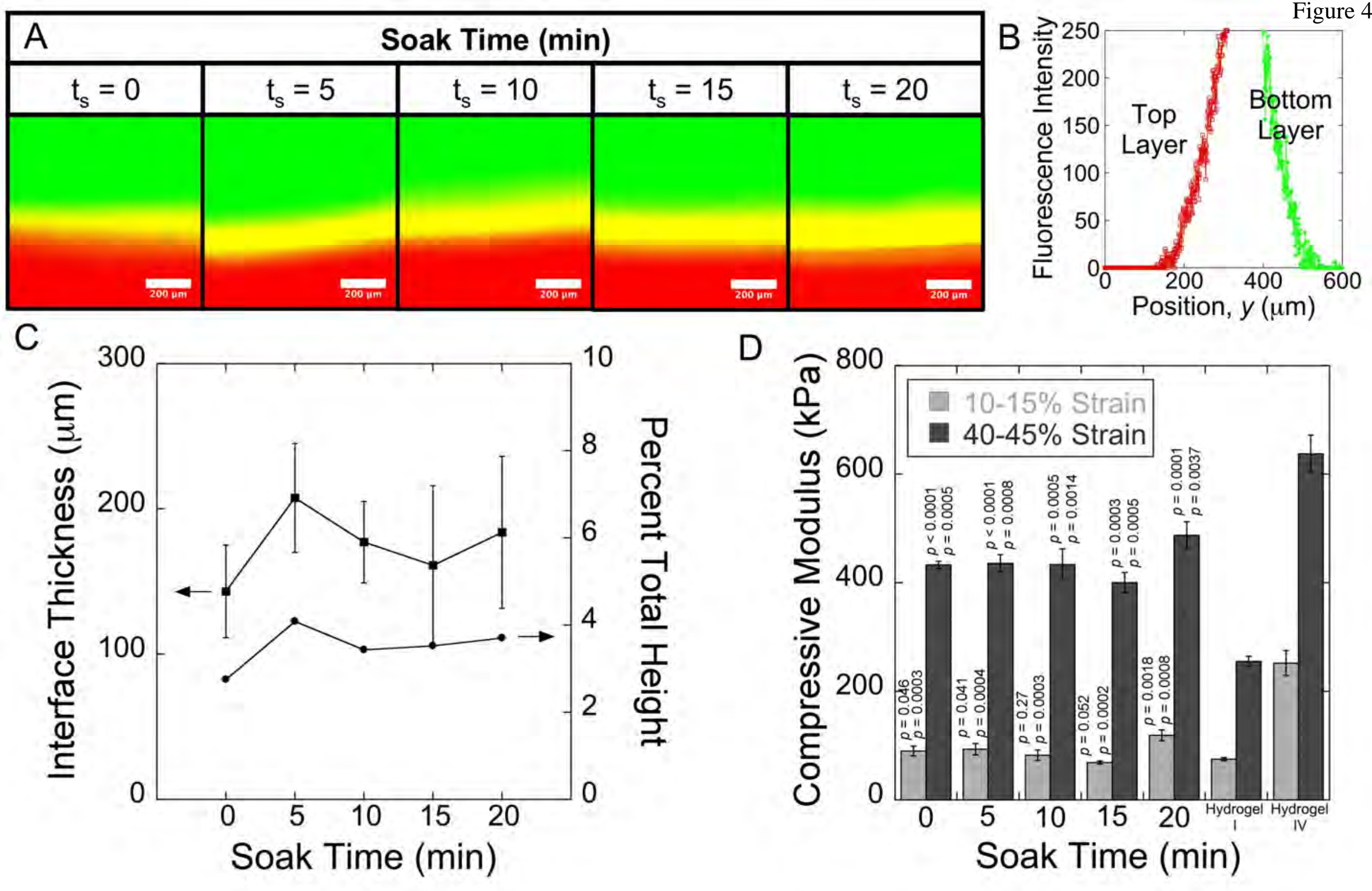



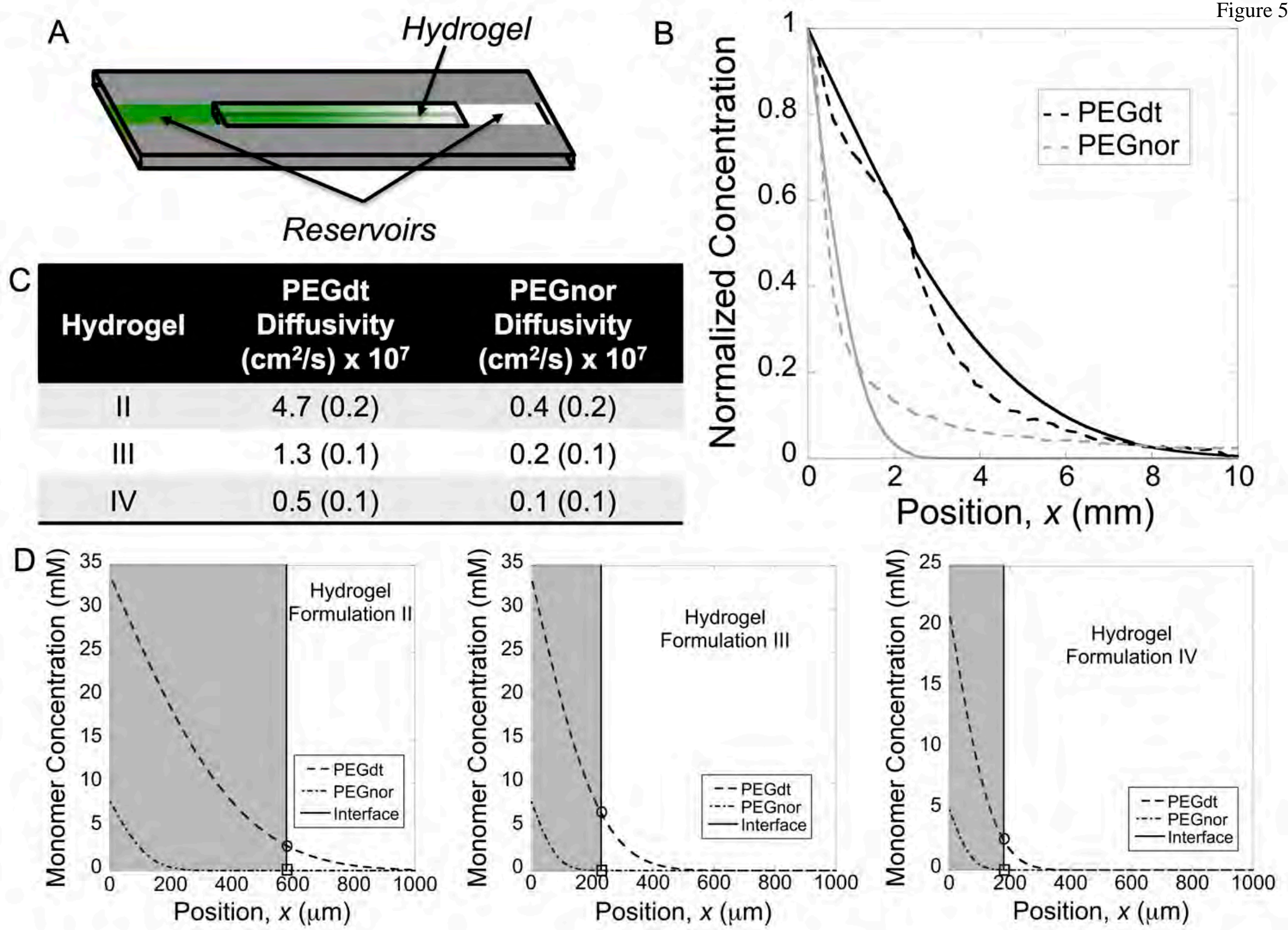


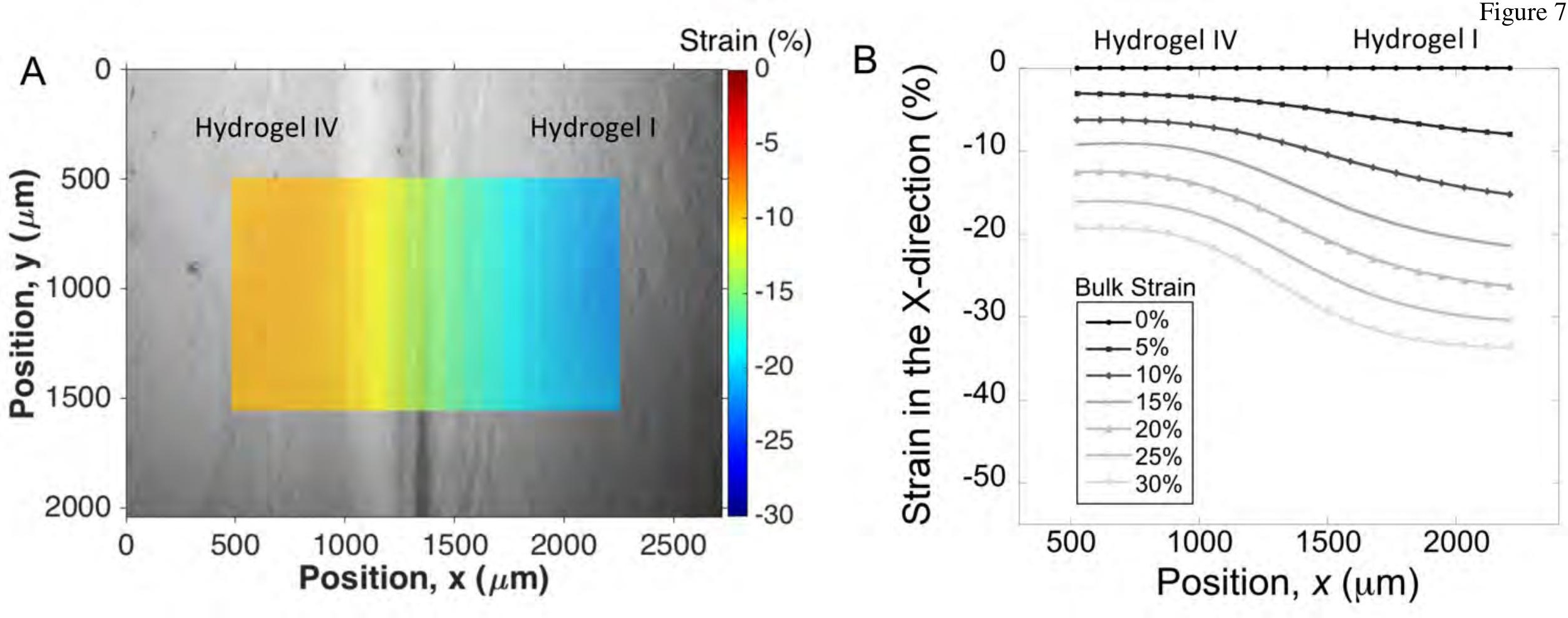

